\title{
Multi-Periodic Photonic Hyper-Crystals: Volume Plasmon Polaritons and the Purcell Effect
}

Babicheva, Viktoriia; lorsh, I. V.; Orlov, A. A.; Belov, P. A.; Lavrinenko, Andrei; Andryieuski, Andrei; Zhukovsky, Sergei

\section{Published in:}

Proceedings of 2014 Conference on Lasers and Electro-Optics (CLEO)

Publication date:

2014

Document Version

Publisher's PDF, also known as Version of record

Link back to DTU Orbit

Citation (APA):

Babicheva, V., Iorsh, I. V., Orlov, A. A., Belov, P. A., Lavrinenko, A., Andryieuski, A., \& Zhukovsky, S. (2014). Multi-Periodic Photonic Hyper-Crystals: Volume Plasmon Polaritons and the Purcell Effect. In Proceedings of 2014 Conference on Lasers and Electro-Optics (CLEO) IEEE. Optics Infobase Conference Papers

\section{General rights}

Copyright and moral rights for the publications made accessible in the public portal are retained by the authors and/or other copyright owners and it is a condition of accessing publications that users recognise and abide by the legal requirements associated with these rights.

- Users may download and print one copy of any publication from the public portal for the purpose of private study or research.

- You may not further distribute the material or use it for any profit-making activity or commercial gain

- You may freely distribute the URL identifying the publication in the public portal 


\title{
Multi-Periodic Photonic Hyper-Crystals: Volume Plasmon Polaritons and the Purcell Effect
}

\author{
V. E. Babicheva, ${ }^{1,2}$ I. V. Iorsh, ${ }^{2}$ A. A. Orlov, ${ }^{2}$ \\ P. A. Belov, ${ }^{2}$ A. V. Lavrinenko, ${ }^{1}$ A. Andryieuski, ${ }^{1}$ and S. V. Zhukovsky ${ }^{1,2, *}$ \\ ${ }^{1}$ DTU Fotonik - Department of Photonics Engineering, Technical University of Denmark, Ørsteds Pl. 343, DK-2800 Kgs. Lyngby, Denmark \\ ${ }^{2}$ National Research University of Information Technology, Mechanics and Optics, Kronverksky pr. 49, St. Petersburg, 197101, Russia \\ *sezh@fotonik.dtu.dk
}

\begin{abstract}
We theoretically demonstrate superior degree of control over volume plasmon polariton propagation and the Purcell effect in multi-period (4-layer unit cell) plasmonic multilayers, which can be viewed as multiscale hyperbolic metamaterials or multi-periodic photonic hyper-crystals. OCIS codes: (160.3918) Metamaterials; (350.4238) Nanophotonics and photonic crystals; (250.5403) Plasmonics.
\end{abstract}

\section{Introduction}

It was recently shown that subwavelength metal-dielectric plasmonic multilayers can be a practical realization of hyperbolic metamaterials (HMMs) - extremely anisotropic media in which the components of the effective permittivity tensor differ in signs (e.g., $\varepsilon_{x, y}<0, \varepsilon_{z}>0$ ) [1]. This gives rise to a hyperbolic dispersion relation, $\left(k_{x}{ }^{2}+k_{y}{ }^{2}\right) / \varepsilon_{z}+k_{z}{ }^{2} / \varepsilon_{x, y}=\omega^{2} / c^{2}$, which in turn results in a variety of new and exotic physical effects [2], including broadband spontaneous emission enhancement (Purcell effect) [3]. It was pointed out that the HMM functionality can be attributed to the existence of "high- $k$ waves" also called volume plasmon polaritons (VPPs) [4,5]. It was further shown that VPPs also exist in a broader class of multilayers compared to HMMs, and persist beyond the HMM regime. It was also demonstrated that any subwavelength metal-dielectric multilayer would simultaneously act as an HMM (for smaller $k$ ) and as a photonic crystal (for larger $k$ ) [6]. This simultaneous presence of two seemingly mutually exclusive paradigms (photonics and metamaterials) in the same class of structures have recently given rise to the term "photonic hyper-crystals" to denote them [7].

Here we generalize the concept of such photonic hyper-crystals (PHC) beyond simple periodicity, introducing, as an example, multi-periodic PHCs. Using the previously introduced multiscale hyperbolic metamaterial concept [8], we show that engineering the structure unit cell allows to tailor the VPP propagation in the wavevector space. By this route, analogs of various photonic structures, such as Bragg mirrors or Fabry-Pérot interferometers, can be introduced for VPPs in HMMs, opening up an additional degree of freedom in controlling light-matter interaction at the nanoscale. As an example, we demonstrate that varying the parameters of a PHC Fabry-Pérot nanocavity containing a dipole emitter can be used to change its Purcell factor by many orders of magnitude.

\section{Multi-periodic photonic hyper-crystals}

As a generalization of a conventional HMM or PHC, we consider a periodic multilayer made of a metal with permittivity $\varepsilon_{m}$ and a dielectric with permittivity $\varepsilon_{d}$, respectively, where the unit cell contains four layers instead of the normal two. The unit cell comprises a pair of metal layer with thickness $d_{m \mathrm{~A}}$ and a dielectric layer with thickness $d_{d \mathrm{~A}}$ stacked with a pair of metal layer with thickness $d_{m \mathrm{~B}}$ and a dielectric layer with thickness $d_{d \mathrm{~B}}(\mathrm{see}$ Fig. 1a). We further assume that $d_{m \mathrm{~A}}+d_{d \mathrm{~A}}=d_{m \mathrm{~B}}+d_{d \mathrm{~B}} \equiv d$ and introduce $\rho_{\mathrm{A}}=d_{m \mathrm{~A}} / d$ and $\rho_{\mathrm{B}}=d_{m \mathrm{~B}} / d$; when $\rho_{\mathrm{A}}=\rho_{\mathrm{B}}$, the introduced "multi-periodic" structure reduces to the conventional PHC. If one regards the first bilayer in the unit cell as A, and the second bilayer as $\mathrm{B}$, then the unit cell can be denoted $\mathrm{AB}$ and the whole structure is a periodic repetition $(\mathrm{AB})^{N}$.

The dispersion diagram of such a structure with an infinite number of periods $(N=\infty)$, calculated using Bloch's theorem, is shown in Fig. 1b. Compared to the conventional HMM, we see that the VPP band is split into two subbands, and a stop band is formed between them. This stop band is akin to a photonic band gap in conventional 1D photonic crystals, which can be seen in the wave vector dependence of reflection coefficient, $\operatorname{Im} R\left(k_{x}\right)$ in the evanescent wave domain $\left(k_{x}{ }^{2}>\varepsilon_{d} \omega^{2} / c^{2}\right)$ in Fig. 1c. It is expected that VPP waves, normally propagating, become evanescent inside the stop band; this is directly confirmed by the electric field distribution inside the structure (Fig. 1d). Hence we have shown that the multi-periodic PHC acts as a Bragg mirror for VPPs in the high- $k$ region.

If we stack the structure $(\mathrm{AB})^{N}$ with its mirror image, the resulting structure $(\mathrm{AB})^{N}(\mathrm{BA})^{N}$ will have a plasmonic analog of a Fabry-Pérot cavity in the middle (Fig. 2a). Hence it is expected that such a nanocavity will feature a narrow "cavity state" inside the VPP stop band where the fields will be localized inside the cavity. This is confirmed in Fig. 2 by showing the reflection coefficient (Fig. 2b) and field distribution (Fig. 2c). It can be seen that the cavity state can be moved across the VPP stop band by adjusting the cavity thickness. 
(a)

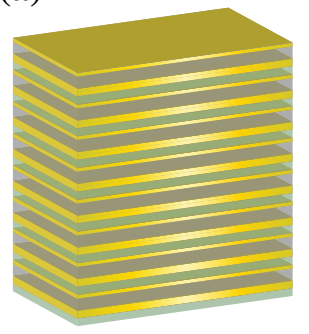

(b)

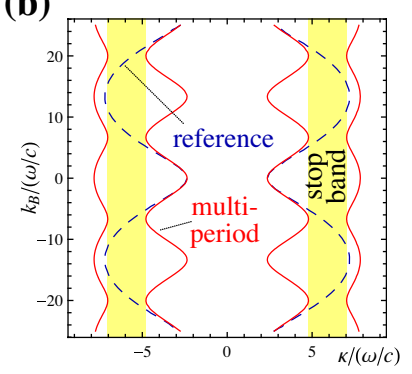

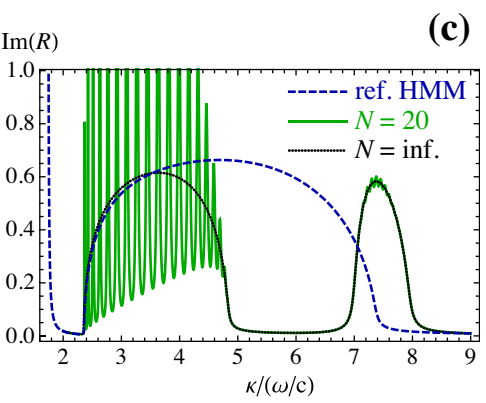

(d)

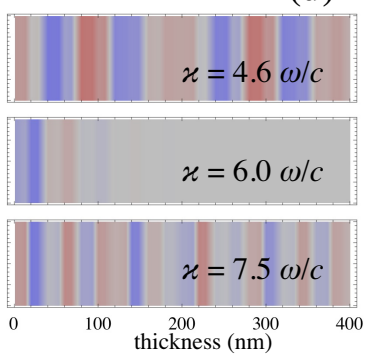

Fig. 1. (a) Schematic and (b) dispersion diagram of an infinite multi-periodic PHC. (c) Reflection coefficient $\operatorname{Im} R\left(k_{x}\right)$ and (d) field maps at values of $k_{x}$ below, inside, and above the stop band. The structure is made of silver and epoxy with $\rho_{\mathrm{A}}=0.6, \rho_{\mathrm{A}}=0.34$, and $d=20 \mathrm{~nm}$.

When an emitter is embedded into such a cavity, it is expected that light-matter interaction phenomena such as the Purcell effect will be strongly modified by virtue of both HMM-based and photonic crystal based contributions. Indeed, Figure $2 \mathrm{~d}$ demonstrates that the Purcell effect for such an emitter inside the cavity may be extremely large and depends very strongly on the location of the emitter inside the cavity. What is even more interesting, we see that the Purcell factor is greatly affected by changing the cavity width even when the distance between the cavity and the closest metal layer remains constant. Hence the control over the Purcell effect cannot be attributed only to the proximity of a metal layer, but rather to the effect of the nanocavity mediated by the VPPs present in it.

(a)

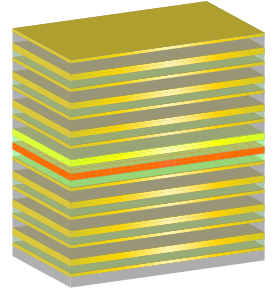

(b)

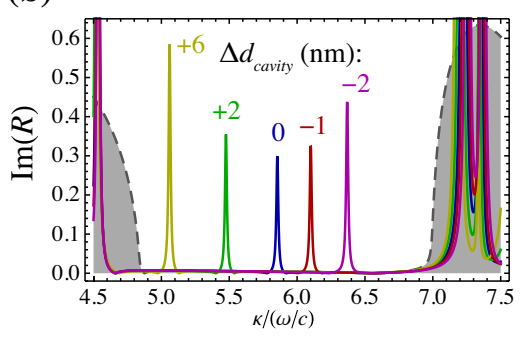

(c)

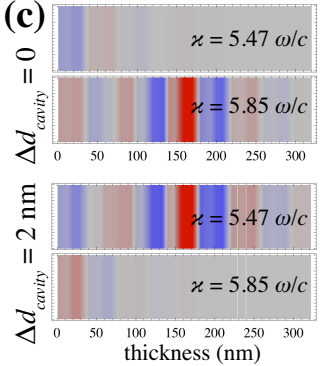

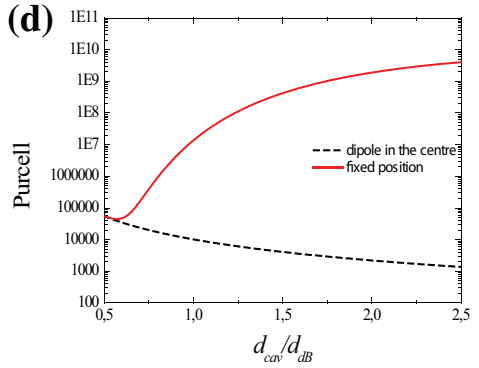

Fig. 2. (a) Schematic of a Fabry-Pérot cavity based on multi-periodic PHC; (b) Reflection coefficient $\operatorname{Im} R\left(k_{x}\right)$ and (c) field mps at values of $k_{x}$ on and out of resonance peak. (d) Purcell factor of a dipole emitter in a cavity of different width if the emitter location is either fixed to the center of the cavity (dashed line) or fixed at the same distance $0.25 d_{d \mathrm{~B}}$ from the nearest metal layer (solid line). Parameters are the same as in Fig. 1

\section{Conclusions}

To summarize, we have shown that multiscale HMMs, or multi-periodic PHCs, open up a new degree of freedom in tailoring the propagation properties of volume plasmonic waves compared to the conventional HMMs, as exemplified by realizing a Bragg mirror (Fig. 1) and a Fabry-Pérot cavity (Fig. 2). The proposed structures are also shown to affect the light-matter interaction of embedded emitters, modifying its Purcell factor by more than five orders of magnitude. Our results imply that the introduced multi-periodic PHCs transcend the concepts of photonic crystals and metamaterials. Indeed, by considering more sophisticated arrangement of the building blocks A and B than a simple alteration $(\mathrm{AB})^{N}$, one can open up the potential of the extensive knowledge in the field of aperiodic photonic multilayers to be utilized in HMMs and, consequently, PHCs [8], for an even more versatile control of light-matter interaction at the nanoscale for use in future-generation nanophotonic and nanoplasmonic applications.

[1] A. Poddubny, I. Iorsh, P. Belov, and Yu. Kivshar, "Hyperbolic metamaterials," Nature Photonics 7, 948-957 (2013).

[2] M. Noginov, M. Lapine, V. Podolskiy, and Yu. Kivshar, "Focus issue: hyperbolic metamaterials," Opt. Express 21, 14895-14897 (2013).

[3] Z. Jacob, I. I. Smolyaninov, and E.E. Narimanov, "Broadband Purcell effect: Radiative decay engineering with metamaterials," Appl. Phys. Lett. 100, 181105 (2012).

[4] S. Ishii, A. V. Kildishev, E. Narimanov, V. M. Shalaev, and V. P. Drachev, "Sub-wavelength interference pattern from volume plasmon polaritons in a hyperbolic medium," Laser Photon. Rev. 7, 265-271 (2013).

[5] O. Kidwai, S.V. Zhukovsky, J.E. Sipe, "Effective-medium approach to planar multilayer hyperbolic metamaterials: Strengths and limitations," Phys. Rev. A 85, 053842 (2012).

[6] S. Zhukovsky, O. Kidwai, J. Sipe, "Physical nature of volume plasmon polaritons in hyperbolic metamaterials," Opt. Expr. 21, 14982 (2013).

[7] E. E. Narimanov, "Photonic Hyper-Crystals," in Proceedings of PQE-2014, Snowbird, Utah, USA (2014), p. 236.

[8] S. V. Zhukovsky, A. V. Lavrinenko, and J. E. Sipe, "Engineering the propagationof high- $k$ bulk plasmonic wavesin multilayer hyperbolic metamaterials by multiscale structuring," in Proc. SPIE, vol. 8915 (Ottawa, Canada), p. 891512 (2013). 